\title{
Síndrome de Burnout em agentes penitenciários: Uma revisão sistemática sob a perspectiva de gênero
}

\section{Burnout syndrome in correctional officers: a systematic review on the view of gender}

\section{Síndrome de Burnout em trabajadores de cárcel: una revisión sistemática desde la perspectica de género}

\author{
Ana Claudia Braun* \\ Pontifícia Universidade Católica do Rio Grande do Sul - PUC-RS, Porto Alegre, Rio \\ Grande do Sul, Brasil
}

\begin{abstract}
RESUMO
O presente estudo objetivou realizar uma revisão sistemática de artigos sobre a temática da Síndrome de Burnout em agentes penitenciários sob a ótica de gênero. No total, foram selecionados 8 artigos. A maioria dos artigos teve como país de publicação os Estados Unidos da América (EUA), entre os anos de 1994 e 2013, predominância de métodos quantitativos, amostra composta por homens e mulheres, contexto prisional masculino, feminino e misto, assim como a utilização do MBI para mensurar a Síndrome de Burnout. A totalidade de artigos teve como objetivo geral de investigar a Síndrome de Burnout e variáveis associadas em agentes penitenciários. Dentre as dimensões da síndrome de burnout, os resultados gerais não demonstram diferenças significativas, porém, apontam para associação positiva da exaustão emocional e desilusão pelo trabalho com tempo de serviço. A dimensão de despersonalização foi mais elevada em homens. As análises demonstram lacunas na produção científica de gênero na categoria de agentes específicos, uma vez que os artigos explorados trouxeram em seu escopo discussões escassas sobre a temática, considerando em sua maioria gênero exclusivamente como a diferença entre homens e mulheres nas amostras exploradas.
\end{abstract}

Palavras Chave: Síndrome de burnout, agentes penitenciários, gênero.

\begin{abstract}
The present study aimed to realize a systematic review of articles in the thematic of Burnout Syndrome in correctional officers in the view of gender. Were selected 8 articles. Most of the articles had as country of publication United States of America (USA), year of publication between 1994 and 2013, predominance of quantitative design, sample composed by man and women, male correctional context, female and mixed as the utilization of the $\mathrm{MBI}$ to measure the Burnout Syndrome. The totality of articles had as main objective investigate the burnout syndrome and variables associated on corrections officers. Among the dimensions of burnout syndrome, the overall results did not show significant differences, however, suggest a positive association between emotional exhaustion and disappointment at work with
\end{abstract}


service time. The dimension of depersonalization was higher in men. The analyses point to gaps in the scientific production of gender in correctional officers, once that the explored articles brought scarce discussions in the scope about the gender thematic, considering gender as a difference between man and woman in the explored samples.

Key Words: Burnout syndrome, correctional officers, gender.

\section{RESUMEN}

El presente estudio ha buscado realizar una revisión sistemática de artículos sobre el tema de Síndrome de Burnout en trabajadores de cárcel desde la perspectiva de género. En total, 8 artículos seleccionados. La mayoría de los artículos se ha publicado en Estados Unidos, año de publicación entre 1994 y 2013, predominio de métodos cuantitativos, muestra compuesta por hombres y mujeres, prisión de género masculino, femenino y mixto, así como el uso de MBI para medir el Síndrome de Burnout. La totalidad de los artículos destinados a investigar el síndrome de burnout y las variables asociadas en agentes penitenciarios. En las dimensiones del síndrome de burnout, los resultados globales no muestran diferencias significativas, sin embargo, apuntan a una asociación positiva entre el agotamiento emocional y la decepción en el trabajo con la antigüedad. La despersonalización fue mayor en los hombres. Los análisis demuestran deficiencias en la producción científica de género en la categoría de trabajadores de cárcel, pues que los artículos explorados han traído en su esencia escasas discusiones acerca del tema, considerando sobre todo género como la diferencia entre hombres y mujeres en las muestras estudiadas.

Palabras clave: Síndrome de burnout, trabajadores de cárcel, género.

\section{Introdução}

O agente penitenciário é reconhecido como aquele que é responsável pelo desempenho das atribuições de vigilância, custódia e aplicação de medidas restritivas de direitos e de privações de liberdade (Barcinski, Altenbernd, \& Campani, 2014). Aos agentes penitenciários é conferida a árdua missão de (re)educar a pessoa que praticou algum crime e garantir que esta possa viver posteriormente em uma condição social que não coloque a sua vida pessoal e as demais em risco (Rosa, 2012). Porém, ao debruçarmos nosso olhar para as atividades laborais do agente penitenciário, percebe-se que o ofício desenvolvido e os riscos que caracterizam seu ambiente são um dos fatores que tornam esta profissão alvo de altos níveis de estresse (Ministério da Saúde, 2001).

Discorrendo especificamente sobre os fatores estressores da profissão, o agente penitenciário é propenso ao estresse oriundo de diferentes naturezas. Uma delas é emergente do conteúdo relacional entre presos e agentes. A literatura aponta que agentes penitenciários são freqüentemente expostos a agressões físicas e psicológicas em seu local de trabalho. Estas agressões são resultantes do contato direto com as pessoas privadas de liberdade e do fato de serem vistos por estas como responsáveis pela 
manutenção do seu confinamento (Fernandes et al., 2002). Ainda, as características peculiares referentes à dinâmica laboral das instituições prisionais não são favoráveis, tanto no que se refere aos aspectos físicos quanto a aspectos materiais (Tschiedel \& Monteiro, 2013). Em conseqüência deste cenário adverso, agentes penitenciários são suscetíveis à insônia, depressão, paranóia e dependências químicas (Silva, 2009). Por estes motivos, conjecturase que a demanda laboral do agente penitenciário pode causar desgaste e conseqüente exaustão no ambiente de trabalho, tornando a profissão altamente estressante (Nery, 2012).

O agente penitenciário, ao estar envolto em um ambiente de constante tensão e estresse, encontra-se imerso em um local com componentes potencializadores para o desenvolvimento da Síndrome de Burnout (Ministério da Saúde, 2001). A Síndrome de Burnout (SB) é uma síndrome psicológica que surge como resposta crônica aos estressores interpessoais vivenciados no ambiente de trabalho (Harrison, 1999, Gil-Monte, 2005). Esta síndrome atinge principalmente indivíduos que trabalham com pessoas de forma direta, constante e com envolvimento emocional (Maslach, 2003). O modelo teórico de Burnout mais amplamente utilizado na literatura é o centrado na concepção psicossocial de Maslach e Jackson (1981). Neste modelo, a SB é constituída por três dimensões: 1) Exaustão Emocional, caracterizada pelo sentimento de carência em recursos emocionais e geralmente relacionada à sobrecarga de trabalho; 2) Despersonalização, dimensão na qual o trabalhador desenvolve sentimentos negativos em relação às pessoas com as quais trabalha, acarretando atitudes coerentes com estes sentimentos, tais como indiferença e cinismo e 3) Baixa Realização Pessoal, identificada pela autoavaliação negativa do sujeito. Esta avaliação negativa afeta diretamente o autoconceito, autoestima e relacionamentos interpessoais estabelecidos no trabalho.

Os aspectos supracitados nos permitem dimensionar a relevância da temática da SB no ambiente de trabalho prisional. Com isso, percebese a importância de trazer a tona o agente penitenciário como escopo de investigação, uma vez que estudos realizados com estes participantes são escassos (Rosa, 2012; Taets, 2013). Sendo assim, este artigo tem como objetivo trazer uma revisão sistemática da literatura versando sobre a síndrome de Burnout em agentes penitenciários. Além, apresenta-se como interesse discorrer sobre o entendimento do desenvolvimento desta síndrome sob a ótica de gênero. Sabe-se que aspectos relacionados a gênero trazem diferenças significativas no processo de adoecimento de homens e mulheres (Miranda \& Lobato, 2009).

É importante contextualizar que, neste artigo, considera-se gênero como um elemento constitutivo das relações sociais fundadas sobre as diferenças percebidas entre os sexos, sendo uma maneira de 
indicar as construções sociais sobre os papéis próprios a homens e mulheres (Scoot, 1989). Alegamos que assumir uma perspectiva de gênero é adotar uma postura crítica que aponta para novas formas de interrogar e priorizar a questão da diferença e da igualdade entre homens e mulheres, categorias que não são universais (Araújo, 2005). Acreditamos que a adoção de uma perspectiva de gênero é fundamental para evidenciar as particularidades das práticas de agentes penitenciários. É a partir das lentes de gênero que podemos teorizar acerca das diferenças entre 0 trabalho de agentes penitenciários do sexo masculino e feminino, bem como das formas particulares de adoecimento evidenciadas por homens e mulheres desempenhando esta função.

\section{Método}

Nesta revisão, optou-se por utilizar as bases BVS, Pubmed, Pepsic e Psycinfo. A escolha específica das bases de dados selecionadas para a pesquisa levou em consideração que a BVS e a Pubmed alojam produções da saúde em geral. Além disso, a BVS contém artigos indexados em outras bases, tais como LILACS, IBECS, MEDLINE, Biblioteca Cochrane e Scielo permitindo abrangência de busca. A Pepsic e Psycinfo, por sua vez, foram selecionadas por promover de forma ampla visibilidade ao conhecimento das ciências do comportamento e da saúde mental. O processo de busca foi realizado entre fevereiro e março de 2014.

Definiram-se como critérios de inclusão: 1- Ser um artigo 2- Estar escrito em língua portuguesa, espanhola ou inglesa. A exploração de produções iniciou através dos descritores "burnout" AND "agentes penitenciários" AND "gênero" através do método de busca "anyfield", porém nenhuma produção foi encontrada em nenhuma das bases.

Diante desta lacuna, foram utilizados os descritores "burnout" AND "correctional officers" AND "gender". Ao utilizarmos estes descritores, encontramos uma produção na base BVS, três na base PubMed, nenhuma na base Pepsic e 11 na base Psycinfo, totalizando 15 produções. Destas, na base BVS uma publicação foi descartada por não atender o critério de inclusão sobre a língua deescrita.Três produções da base Psycinfo, foram descartadas por serem dissertações. Na base PubMed houve a exclusão de três artigos por se encontrarem repetidos na base Psycinfo. Por final, o total de artigos selecionados para esta revisão foi 8 , todos provenientes da base Psycinfo.

Após a busca de artigos e sua leitura integral, foram definidos os seguintes critérios de classificação: 1- Pais de publicação do estudo; 2- ano de publicação; 3- objetivo geral; 4- delineamento; 5amostra; 6- tipo de instituição prisional estudada; 7-instrumentos 
utilizados; 8- Conclusão e principais achados. Os critérios de classificação foram determinados a partir de discussões realizadas junto a psicólogos especialistas no assunto, focando nos aspectos e objetivos a serem alcançados nesta revisão sistemática.

\section{Resultados}

A seguir, o conteúdo emergente de cada critério de classificação será exposto, sendo divididos em subitens. A divisão visa trazer uma compreensão das categorias mencionadas, assim como inferências sobre as principais características apresentadas.

\subsection{País e ano de publicação}

Os estudos tiveram como países de publicação Estados Unidos (seis artigos), Canadá (um artigo) e Espanha (um artigo). Os anos de publicação dos estudos variaram de 1994 a 2013sendo que, cronologicamente, estão datados em 1994, 1997, 2002, 2003 (dois artigos), 2006, 2011 e 2013. Este resultado evidencia uma concentração geográfica de publicações provenientes dos Estados Unidos e temporalmente espaçadas, uma vez que não se evidenciou um ano específico de publicação com maior concentração.

\subsection{Objetivos}

Como objetivos dos estudos investigados, identificou-se: 1Investigar a prevalência de Burnout e as estratégias de enfrentamento utilizadas por agentes de 10 penitenciárias em uma província Canadense; 2- Descrever os riscos à saúde em profissionais que trabalham em unidades prisionais; 3- Replicar a estrutura fatorial do Maslach Burnout Inventory - MBI e examinar a relação entre SB e variáveis sociodemográficas em agentes penitenciários; 4- Investigar a relação entre assédio moral e a SB em uma unidade prisional; 5Investigar e comparar a relação entre gênero e Burnout em agentes penitenciários de duas prisões estaduais dos EUA, sendo uma masculina e uma feminina; 6- Investigar a relação entre variáveis que levaram a resultados inconsistentes em pesquisas anteriores sobre estresse em agentes penitenciários e integrar mais duas variáveis ao estudo (cargo e satisfação pessoal); 7- Explorar as diferenças de gênero em relação às formas como agentes penitenciários reagem ao estresse ocupacional severo e verificar as diferenças nas estratégias de coping; 8- Investigar a relação entre gênero e estresse relacionado ao trabalho vivenciado por agentes penitenciários. Os objetivos diferem especialmente nas variáveis 
associadas ao Burnout, demonstrando diversidade no escopo de pesquisa dos artigos explorados.

\subsection{Delineamento, amostra e tipo de instituição investigada}

O delineamento utilizado foi predominantemente quantitativo (sete artigos), com incidência de método misto (um artigo). Em todos os artigos, a amostra foi composta por agentes penitenciários homens e mulheres (oito artigos). Especificamente sobre os tipos de unidades prisionais investigadas foram utilizadas amostras comparativas entre penitenciárias masculinas e femininas com agentes de ambos os sexos, trabalhadores de uma prisão de segurança média- máxima masculina e uma exclusivamente feminina com o mesmo nível de segurança (um artigo), amostra de prisão masculina de segurança média com agentes homens e mulheres (dois artigos), amostra proveniente de prisão mista com homens e mulheres, onde não é relatado o nível de segurança da instituição (um artigo), agentes homens e mulheres trabalhadores de uma prisão de segurança máxima, média e mínima (um artigo), amostra comparativa de agentes homens e mulheres alocados em centros penitenciários para jovens e adultos (um artigo) e, também, foram encontrados artigos com o tipo de instituição prisional não referido (dois artigos). Este resultado demonstra a prevalência de artigo com método semelhante e totalidade de amostras mistas, contextualizando homens e mulheres sob a mesma ótica de análise. Em relação ao tipo de instituição prisional investigada, não há a mesma homogeneidade dos dois itens anteriores, indicando a possibilidade de resultados divergentese influenciados pelo contexto laboral nos artigos avaliados.

\subsection{I nstrumentos}

O instrumento quantitativo utilizado para mensurar as dimensões de SB foi, na maioria dos estudos, o Maslach Burnout Inventory - MBI (seis artigos), havendo ainda a utilização de instrumentos como a Burnout Scale on Exhaustion and Disengagement (um artigo) e o MBI-HSS (um artigo). Este resultado ratifica a prevalência do MBI para a investigação da SB, uma vez que é proveniente do modelo de Maslack e Jackson (1981), utilizado amplamente na literatura internacional.

\subsection{Conclusão e principais achados}

A seguir, será apresentada a conclusão e os principais achados dos artigos desta revisão. Para fins de melhor entendimento dos aspectos convergentes, os resultados explorados serão compilados em seus 
achados similares e concentrados especificamente nas dimensões de Burnout, uma vez que estas constituem a temática central do artigo. Avaliando de forma geral a SB, os resultados da pesquisa de Hurst e Hurst (1997) realizada em penitenciárias masculinas e femininas de segurança média demonstraram poucas diferenças nas dimensões de SB investigadas. Comparando as normas do MBI, homens e mulheres apresentaram índices elevados na dimensão de despersonalização e baixos índices na dimensão de realização pessoal, indicando um alto nível de estresse no trabalho. Homens e mulheres não pontuaram de forma significativamente diferente na dimensão de exaustão emocional, apesar de os sujeitos indicarem sentimentos de estarem emocionalmente exaustos em seu trabalho. Estudo de Carlson, Anson e Thomas (2003), ao evidenciar resultados gerais obtidos na amostra composta por agentes de ambos os sexos de penitenciárias masculinas e femininas, revela que homens e mulheres possuem índices elevados de Burnout. Gould, Watson, Price e Valliant (2013) relataram que agentes penitenciários de ambos os sexos apresentam níveis elevados na dimensão de exaustão emocional, despersonalização e níveis de realização pessoal medianos.

Relacionando níveis de estresse geral e Burnout com gênero, estudo de Gross et al (1994) aponta que somente $10 \%$ da variabilidade encontrada no estresse entre homens e mulheres pode ser atribuída a fatores relacionados às diferenças de gênero, inferindo que ambos os sexos experimentam níveis similares elevados de estresse e Burnout. Morse et al. (2011) ratificam que estes fatores de estresse experienciados por ambos os sexos são associados à saúde de forma negativa, podendo levar a doenças crônicas físicas e psicológicas. No estudo dos mesmos autores, foram identificadas como fonte de estresse questões administrativas e burocráticas, tais como os longos plantões realizados pelos agentes, e questões de cunho pessoal, como a privação do convívio social junto a familiares.

Referente à dimensão de exaustão emocional, o tempo de experiência foi correlacionado de forma positiva com exaustão emocional e redução de realização pessoal. Morgan, Van Haveren e Pearson (2002) evidenciaram menor exaustão emocional em agentes que trabalhavam há menos de um ano na função. Estudo de Hernández-Martín et al. (2006) associou o incremento da exaustão emocional ao grupo de sujeitos com menos de 15 anos de experiência na função. Estudo de Hurst e Hurst (1997) não constatou diferenças significativas em exaustão emocional na comparação entre homens e mulheres, sendo que ambos apresentaram níveis elevados similares.

$\mathrm{Na}$ pesquisa de Savicki, Cooley e Gjesvold (2003) os escoresda dimensão exaustão emocional foram elevadosquando associados à maior pressão no trabalho e menor recompensas por parte da supervisão. Os mesmos autores apontam que, quando a exaustão emocional é elevada em mulheres, a mesma é associada com o 
assédio e a pressão elevada no ambiente de trabalho. Em contraponto, a análise multivariada dos resultados da pesquisa realizada por Carlson, Anson e Thomas (2003) ratifica que gênero não é um preditor significativo para esta dimensão, sendo que neste estudo a experiência anterior militar e o treinamento para o estresse foram fatores associados à redução na dimensão de exaustão emocional em agentes.

Especificamente sobre a dimensão de despersonalização, estudo de Carlson, Anson e Thomas (2003), Savicki, Cooley e Gjesvold (2003) e de Gould et al. (2013), conduzidos com amostras de homens e mulheres, apresentaram resultados similares ao estudo de Morgan, Van Haveren e Pearson (2002) e Gross et al (1994), sendo que em ambas pesquisas homens, quando comparados com mulheres, demonstraram maior pontuação nesta dimensão de Burnout. Ainda, no estudo de Carlson, Anson e Thomas (2003) a maior despersonalização em homens foi atribuída aos agentes de menor idade, quando comparados com agentes de maior idade. Da mesma forma, estudo de Hernández-Martín et al (2006) atribuiu a maior despersonalização a agentes com menos de 40 anos de idade e menos de 15 anos de experiência na função. Morgan, Van Haveren e Pearson (2002) associaram a despersonalização ao tempo de trabalho, sendo que agentes que trabalham há menos de 1 ano na função de agente demonstram menores índices nesta dimensão. Estudo de Savicki, Cooley e Gjesvold (2003) correlacionou de forma positiva à dimensão de despersonalização em homens quando atrelada a altas demandas de trabalho e baixo reconhecimento por parte da supervisão. Para mulheres, o mesmo estudo associou a elevação desta dimensão com o assédio e estilo coercitivo de gestão.

Em contraponto aos resultados supracitados, estudo de Hurst e Hurst (1997) apontou índices elevados de despersonalização similares em homens e mulheres. Estudos de Gould et al. (2013) e Morgan, Van Haveren e Pearson (2002) não demonstraram diferenças nas médias encontradas na dimensão de exaustão emocional na comparação entre homens e mulheres.

Em relação à dimensão realização pessoal estudo de Hurst e Hurst (1997) identificou baixos índices nesta dimensão. Igualmente, Morgan, Van Haveren e Pearson (2002) não identificaram diferenças significativas entre homens e mulheres nesta dimensão. Já em estudo de Carlson, Anson e Thomas (2003) agentes do sexo feminino demonstraram maior realização pessoal quando comparadas com agentes do sexo masculino. Outro fator ligado à realização pessoal foi o tempo de experiência. Estudo de Carlson, Anson e Thomas (2003) evidenciaram que quanto maior o tempo de experiência do agente menor a sua realização pessoal. Hernández-Martín et al (2006) afirma que agentes com menos de 15 anos de experiência apresentaram menor realização pessoal, enquanto Morgan, Van 
Haveren e Pearson (2002) apontam que agentes com menos de um ano de trabalho demonstraram maior realização pessoal.

\section{Discussão sob a ótica de gênero}

Inicialmente, ressalta-se que a discussão que segue tem como objetivo contemplar a ampliação do entendimento dos resultados dos artigos selecionados a luz de teorias de gênero. Os artigos que complementam a discussão não elucidam diretamente estudos de Burnout, porém, contemplam a categoria de gênero como parte de um desenvolvimento social, diferenciado para homens e mulheres. Os artigos complementares tornam-se importantes na medida em que discutem gênero no ambiente profissional e potencializam 0 entendimento do Burnout. Assim, formam-se interseções possíveis para a explicação e compreensão de resultados alcançados junto aos artigos selecionados nesta revisão.

De maneira geral os resultados dos artigos selecionados apontam para diferenças entre homens e mulheres no que se refere ao desenvolvimento da SB. No entanto, percebe-se que nas discussões dos resultados, os autores não se engajam em um discurso a partir da perspectiva de gênero. Inferimos que há diferenças específicas entre a realidade percebida entre homens e mulheres dentro do contexto laboral penitenciário. Estas diferenças são notórias nos processos relacionais estabelecidos e fazem sentir seus reflexos de formas distintas na saúde ocupacional de agentes homens e mulheres uma vez que, como afirma Rohlfs (1999), a atuação social assumida por homens e mulheres é relevante para a explicação de sua condição de saúde.

Para o entendimento de determinadas peculiaridades precisamos nos referir às formas diferenciadas de envolvimento em atividades laborais de homens e mulheres, bem como compreender seus processos particulares de socialização e de adoecimento. A partir desta perspectiva, a discussão que segue objetiva compreender as principais conclusões e principais achados acima elencados sob uma perspectiva de gênero. Em suma, trata-se de entender que a experiência de homens e mulheres trabalhadores do sistema prisional traz as marcas das formas como homens e mulheres se relacionam e desenvolvem suas atividades na sociedade mais ampla.

Em relação ao desenvolvimento da SB, pode-se pressupor que homens e mulheres tendem a apresentar diferentes fontes de estresse. Parte destas diferenças pode estar centrada na percepção acerca do contexto prisional. Em decorrência de o ambiente prisional ser predominantemente masculino (Miyamoto \& Khroling, 2012) a mulher tende a se esforçar de forma significativa para alcançar as expectativas deste ambiente de trabalho, almejando ser percebida 
tão efetiva quanto os homensno ambiente prisional (Withrow, 1992). Esse fato é de maior alcance, uma vez que Belo e Camino (2012) afirmam que, apesar de todas as mudanças ocorridas socialmente, ainda há um apego a ideologia que mulheres são menos capacitadas do que homens para funções específicas, como a de agente penitenciário.

Ao entendermos a constituição da subjetividade feminina primordialmente atrelada ao ato de cuidar do outro (Marcondes, 2013), podemos conjecturar que a necessidade de se posicionar de forma autoritária frente aos sujeitos detidos seja altamente desgastante para as mulheres, elevando seus níveis de exaustão emocional. No desempenho das funções de controle e vigilância as agentes penitenciárias se apropriam de características culturalmente associadas ao masculino, tais como a agressividade e o pragmatismo (Barcinski, Altenbernd, \& Campani, 2014). Devido ao fato de mulheres serem frequentemente selecionadas e estimuladas a funções definidas como femininas (Purvanova \& Muros, 2010), 0 ambiente prisional pode ser considerado adverso aos estereótipos sociais de inserção da mulher. Fernandes et al. (2002) apontam que a ênfase feminina no cuidado, em detrimento da força, pode resultar em uma menor capacidade de reagir às tensões próprias do trabalho, o que geraria maior pressão e desgaste para as agentes do sexo feminino.

Neste sentido, acreditamos que a necessidade de se provar efetiva no ambiente de trabalho se expresse pelas tentativas de desenvolver atitudes socialmente associadas ao masculino, tais como exercer a autoridade frente aos indivíduos privados de liberdade. Portanto, agentes do sexo feminino se vêem obrigadas a transgredir as normas de gênero no que diz respeito às atitudes e comportamentos legitimados socialmente para homens e mulheres. A literatura PSI aponta que, de forma geral, o ingresso de mulheres em territórios dominados por homens é caracterizado pelo alinhamento destas à norma masculina (Yannoulas, 2013). Refere Guimarães (2004) que com a intensificação das mulheres junto ao mercado de trabalho, criou-se um amplo leque de expectativas sobre os padrões de inclusão destas mulheres no espaço laboral que ocupam. Profissionais do sexo feminino que executam suas atividades em ambientes predominantemente masculinos tendem a exclusão caso não haja conformidade a características masculinizantes (Belo e Camino, 2012). Em contraponto, homens tendem a vivenciar o ambiente prisional de forma menos tensa e estressante em conseqüência da identificação da posição do trabalho com características masculinas de dominação, controle e força (Miyamoto \& Khroling, 2012).

Uma das diferenças entre homens e mulheres oriundas dos processos relacionais no trabalho foi verificada na dimensão de despersonalização. Esta dimensão é diretamente ligada a atitudes 
negativas, indiferença e cinismo em relação às pessoas com quem se relaciona no trabalho (Maslach \& Jackson, 1981). O fato de que os homens apresentam expectativas elevadas, no que diz respeito ao seu desempenho profissional e expectativas de sucesso (Schaufeli \& Greenglass, 2001), pode colaborar para a maior competitividade, relegando as questões relacionais para o segundo plano. Caetano e Neves (2009) afirmam que homens possuem maior habilidade de separação entre a esfera do trabalho e pessoal sendo mais objetivos, assertivos e autoritários frente às demandas laborais. Assim, a indiferença com os clientes/indivíduos atendidos e a ausência de vínculos relacionais afetivos pode emergir como propulsor da despersonalização em agentes penitenciários homens. Ratifica Welzer-Lang (2001) que homens, para serem verdadeiros homens, devem combater aspectos que podem igualá-los às mulheres, tais como a manifestação de sentimentos.

Ainda, uma vez que o trabalho na prisão prioriza características tipicamente masculinas não ligadas ao feminino, homens possivelmente tendem a corresponder de forma mais efetiva às expectativas de controle e vigilância das pessoas em situação de prisão. Como resultado desta interação, que pressupõe o afastamento afetivo entre agentes e presos, os homens podem desenvolver atitudes negativas e indolentes, fazendo com que esta os índices na dimensão de despersonalização sejam mais elevados em agentes do sexo masculino.

No que diz respeito aos menores índices de despersonalização em mulheres, Yannoulas (2013) afirma que às mulheres são associadas características de docilidade e capacidade de conciliação. A característica de docilidade pode ser entendida como parte da construção da identidade social da mulher, uma vez que, como afirma Araújo e Scalon (2005) historicamente foram se formando "tipos de trabalho" sendo que homens foram designados a questões produtivas e mulheres ao cuidado e diligência. De forma similar, Marcondes (2013) afirma que a existência social feminina realiza-se na medida em que há a ação de cuidar do outro. Ainda, no processo de socialização são designadas às mulheres funções que enfatizam o cuidado (Miyamoto \& Krohling, 2012). Podemos inferir, portanto, que às mulheres seja socialmente permitida a maior proximidade com os indivíduos no ambiente de trabalho, bem como a demonstração de afeto e cuidado com os mesmos. Ratifica Maslach e Jackson (1985) que a expressão de emoções é socialmente estimulada em mulheres e Ihes confere o consentimento da maior manifestação de sentimentos. No contexto prisional, tal permissão para a expressão do afeto pode se traduzir em maior proximidade emocional com os sujeitos privados de liberdade e, conseqüentemente, menor despersonalização das mulheres em comparação aos homens agentes. 
Outro resultado encontrado nesta revisão refere-se à realização pessoal, onde agentes que trabalham em centros penitenciários femininos apresentam maiores índices nesta dimensão e menor exaustão emocional. Ao pensarmos sobre a questão de realização pessoal no trabalho e diferenças na exaustão emocional no contexto prisional, a mulher vê-se impossibilitada de se relacionar de forma plena e, tal fato, produz uma ruptura de identidade. Sendo assim, a mulher torna-se não situadas no contexto em que está inserida devido à impossibilidade de exercer características relacionais atreladas ao feminino (Cunha, 1994). Cremos que no contexto prisional mulheres não encontram os recursos necessários para exercer a sua feminilidade, relacionar-se e exercer papeis tradicionalmente associados ao feminino, como de mãe e esposa. Essa questão torna-se um limitador, a saber, que especificidades relativas ao cotidiano de trabalho das mulheres não encontram espaço adequado para a sua manifestação, como relata Barros (2008), havendo dificuldade de ver e dar visibilidade aos aspectos e questões específicas das mulheres em seus locais de trabalho. Por esse motivo, conjecturamos que, em prisões femininas, por necessidade de ser novamente situável, mulheres tendem a demandar das agentes o conteúdo relacional, e necessário, ao gênero feminino. É possível que nas prisões femininas os agentes sejam mais demandados no sentido de estabelecerem contatos mais emocionais e pessoais com as mulheres presas.

Apesar das diferenças significativas no processo de socialização e de inserção no mercado de trabalho entre homens e mulheres, alguns estressores no trabalho de agente penitenciário são inerentes à profissão. De forma indiscriminada, os presos tendem a questionar constantemente a autoridade do agente penitenciário e hostilizar os vínculos estabelecidos (Fernandes et al., 2002). Portanto, pelas características peculiares ao trabalho do agente penitenciário, a elevação da dimensão de exaustão emocional é identificada de forma similar em agentes de ambos os sexos. O fato é potencializado por ambos vivenciarem situações laborais e demandas institucionais igualmente adversas.

Cabe ressaltar, ainda, que em grande parte dos artigos selecionados a temática de gênero foi explorada a partir de análises estatísticas e discussões que propiciaram resultados apoiados na diferença entre os sexos, não contextualizando de forma ampla as diferenças advindas do processo de socialização ou de inserção profissional de homens e mulheres, por exemplo. Portanto, de maneira geral os artigos adotaram gênero como uma variável explicativa das diferenças entre homens e mulheres, seja nas dimensões da SB ou na forma de envolvimento e percepção do trabalho de agente penitenciário.

Por fim, ampliando a discussão para aspectos relativos ao método das pesquisas investigadas, cabe destacar que os resultados desta 
revisão evidenciaram ausência de artigos com delineamento qualitativo, bem como de discussões que considerem de forma aprofundada a compreensão das relações de gênero e suas implicações para a saúde no contexto prisional. Ainda, notou-se a ausência de artigos que estejam centrados no contexto prisional brasileiro, uma vez que sabemos das peculiaridades sociais e institucionais que emergem de diferentes regiões e países. Este resultado foi demonstrado através do número de produções encontradas conforme os critérios de seleção determinados, não contemplando publicações nacionais acerca do tema.

\section{Considerações finais}

O estudo teve como objetivo apresentar uma revisão sistemática de artigos que envolvam a temática do desenvolvimento da SB em agentes penitenciários sob uma perspectiva de gênero. Apesar da grande relevância, a produção de artigos e pesquisas que têm como objetivo compreender o desenvolvimento da SB em agentes penitenciários é exígua (Rosa, 2012). Por esse motivo, visualizou-se a necessidade de investigações científicas e futuras intervenções da saúde ocupacional de agentes que possam levar em conta as diferenças e similaridades de gênero neste contexto.

Ainda, apontamos para novas pesquisas nesta área de investigação, principalmente que considerem as diferenciações de características peculiares a homens e mulheres e tragam em seu escopo outros importantes marcadores como classe, raça e nacionalidade, que devem ser explorados junto à questão de gênero. Sugere-se a exploração de diferentes materiais sobre a temática, incluindo teses, livros e dissertações em novas pesquisas, assim como a exploração de novas bases para o incremento de investigações e achados nesta área de produção científica.

\section{Referências}

Araújo, M. F.(2005). Diferenças e igualdades nas relações de gênero. Psicologia Clínica, 17(2), 41-52.

Araújo, C., \& Scalon, C. (2005). Percepções e atitudes de mulheres e homens sobre a conciliação entre família e trabalho no Brasil. Gênero, família e no Brasil. Rio de Janeiro: Fundação Getúlio Vargas.

Barcinski M., Altenbernd, B., \& Campani, C. (2014). Entre vigiar e punir: Ambiguidades e contradições do agente penitenciário. Ciência e Saúde Coletiva, 19(7), 2245-2254. 
Barros, A. M. (2008). Cidadania, relações de gênero e relações de trabalho. Revista do Tribunal Regional do Trabalho, 47(77), 6783.

Belo, R.P., \& Camino, L.(2012) Trabalho e gênero: elaborações discursivas sobre os papéis sociais. Cadernos de Psicologia Social e do Trabalho, 15(2), 271-286.

Caetano, E., \& Neves, C.E. (2009). Relações de gênero e precarização do trabalho docente. Revista HISTEDBR On-line, (n. especial), 251263.

Carlson, J.R., Anson, R. H., \& Thomas, G. (2003). Correctional officer burnout and stress: Does gender matter? The Prison Journal, 83(277), 277-286.

Cunha, M. I. (1994). Malhas que a reclusão tece: Questões de identidade numa prisão feminina. Lisboa: Centro de Estudos Judiciários.

Fernandes, R. C. P., Silvany Neto, A. M. S., Sena, G. M., Leal, A. S., Carneiro, C. A. P., \& Costa, F. P. M. (2002). Trabalho e cárcere: um estudo com agentes penitenciários da Região Metropolitana de Salvador, Brasil. Cadernos de Saúde Pública, 18(3), 807-816.

Gil-Monte, P. R. (2005). El Síndrome de Quemarse por el Trabajo. Madrid: Pirâmide.

Gould, D. D., Watson, S., Price, R. \& Valliant, P. (2013). The relation between burnout and coping in adult and young offender center correctional officers: an exploratory investigation. Psychological Services, 10(1), 37-47.

Gross, G. R., Larson, S. J., Urban, G. D., \& Zupan, L. L. (1994). Gender differences in occupational stress among correctional officers. American Journal of Criminal Justice, 18(2), 219-234.

Guimarães, N. A. (2004). Gênero e trabalho. Revista Estudos Feministas, 12(2), 145-146.

Harrison, W. D. (1999). A social competence model of burnout. In B. A. Farber (Ed.), Stress and burnout in the human services professions (pp. 29-39). New York: Pergamon Press.

Hernández-Martín, L., Fernández-Calvo, B., Ramos, F., \& Contador, I. (2006). El síndrome de burnout en funcionarios de vigilancia de un centro penitenciário. International Journal of Clinical and Health Psychology, 6(3), 599-611.

Hurst, T. E., \& Hurst, M. M. (1997).Gender differences in mediation of severe occupational stress among correctional officers. American J ournal of Criminal J ustice, 22(1), 121-137

Marcondes, M. M. (2013). O cuidado na perspectiva de divisão sexual do trabalho: contribuição de estudos sobre a feminização no mundo do trabalho. In S. C. Yamoullas (Org.). Trabalhadoras: análise da feminização das ocupações e profissões. Brasília: Abaré. 
Maslach, C. (2003). Burnout: The cost of caring. Cambridge: Malor Books.

Maslach, C., \& Jackson, S. E. (1981). The measurement of experienced Burnout. Journal of Occupational Behavior, 2, 99113.

Maslach, C., \& Jackson, S. E. (1985). The role of sex and family variables in burnout. Sex Roles, 12(7/8), 837-851.

Ministério da Saúde (2001). Doenças relacionadas ao trabalho: Manual de procedimentos para os serviços de saúde. Brasília, DF: MS.

Miranda, D., \& Lobato, S. (2009). Processos de adoecimento ligados a gênero: uma história de (des)valorização dos múltiplos papéis femininos. Retrieved from: http://artigocientifico.uol.com.br/uploads/artc_1206559548_43.p df

Miyamoto, Y., \& Krohling, A. (2012). Sistema prisional brasileiro sob a prespectiva de gênero: invisibilidade e desigualdade da mulher encarcerada. Direito, Estado e Sociedade, 40, 223-241.

Morgan, R. D., Van Haveren, R. A., \& Pearson, C. A. (2002). Correctional officer's burnout: Further Analyses. Criminal Justice and Behavior, 29(2), 144-160.

Morse, T., Dussetschleger, J., Warren, N., \& Cherniack, M. (2011). Talking about health: Correctional employees'assessments of obstacles to healthy living. Journal of Environmental Medicine, 53(9), 1037-1045.

Nery, T. R. A. (2012). Da ética à poética do ser servidor penitenciário. Porto Alegre: Companhia Rio-Grandense de Artes Gráficas.

Puranova, R. K., \& Muros, J. P. (2010). Gender differences in burnout: A meta-analysis. Journal of Vocational Behavior, 77, 168-185.

Rohlfs, D. (1999). La perspectiva de género en el estudio de las diferencias y desigualdades en salud. I Jornada de la Red de Médicas y Profesionales de la Salud, (2): 12-13.

Rosa, L. R. (2012) Relato de Pesquisa: Estresse e Qualidade de Vida dos Servidores Penitenciários. In: T. R. A., Nery (Org.). Da ética à poética do ser servidor penitenciário. Porto Alegre: Companhia Rio-Grandense de Artes Gráficas.

Savicki, V., Cooley, E., \& Gjesvold, J. (2003). Harrasment as a predictor of job burnout in correctional officers. Criminal Justice and Behavior, 30(602), 602-619.

Scoot, J. (1989). Gender: a useful category of historical analyses. Gender and the politics of the history. New York, Columbia University Press.

Schaufeli, W. B., \& Greenglass, E. R. (2001). Introduction to special issue on burnout and health. Psychology and Health, 16, 501-510. 
Disponível

em:

http://userpage. fuberlin.de/ schuez/folien/schaufeli. pdf.

Acessado em: 10/04/2013.

Silva, J. M. C. (2009). Adversidades no sistema penitenciário. Disponível em: http://www.sifuspesp.org.br/index.php/component/content/579.h tml?task=view. Acesso em 30/01/2014.

Taets, A. R. F.(2013). Em trânsito: o cotidiano de algumas agentes de segurança penitenciária no estado de São Paulo. Mediações, 18(2), 246-259.

Tschiedel, R. M., \& Monteiro, J. K. (2013). Prazer e Sofrimento no trabalho das agentes de segurança penitenciária. Estudos de Psicologia, 18(3), 527-535.

Welzer-Lang, D. (2001). A construção do masculino: dominação das mulheres e a homofobia. Estudos Feministas, 9, 460-482.

Withrow, P. (1992). Workplace reality: Women staff tell it like it is. Corrections Today, 5, 88-92.

Yannoulas, S, C.(2013) Trabalhadoras: análise da feminização das profissões e ocupações. Brasília: Abaré.

\section{Endereço para correspondência}

Ana Claudia Braun

Pontifícia Universidade Católica do Rio Grande do Sul

Av. I piranga, 6681, Partenon, CEP 90619-900, Porto Alegre - RS, Brasil

Endereço eletrônico: anaclaudiabraun@hotmail.com

Recebido em: 20/08/2015

Reformulado em: 21/12/2015

Aceito para publicação em: 24/02/2016

\section{Notas}

* Psicóloga (Universidade Feevale) e Especialista em Psicologia Organizacional (ESADE). Mestre e Doutoranda em Psicologia Social (PUCRS). Docente no Unilasalle Canoas - RS. 